\section{D) Check for updates}

Cite this: Food Funct., 2020, 11 6807

Received 1st March 2020, Accepted 11th July 2020

DOI: 10.1039/d0fo00537a

rsc.li/food-function

\title{
The effects of betalain-rich cacti (dragon fruit and cactus pear) on endothelial and vascular function: a systematic review of animal and human studies $\uparrow$
}

\author{
Alex Cheok, (D) ${ }^{a}$ Trevor W. George, ${ }^{a}$ Ana Rodriguez-Mateos (D) *a and \\ Paul W. Caton ${ }^{a, b}$
}

\begin{abstract}
Dragon fruit (Hylocereus) and cactus pear (Opuntia) are cacti species that have been widely used globally as a reliable source of food as well as traditional folk remedies. They have become of scientific interest recently due to their high levels of bioactive phytochemical compounds, in particular betalains. Earlier systematic reviews have explored the impact of supplementation of these cactus species on obesity, type-2 diabetes mellitus and cardiovascular risk factors: body weight and composition, serum triglycerides, cholesterol, blood glucose and blood pressure. However, effects on vascular health and endothelial function have yet to be reviewed. In order to address this gap in the literature, a systematic review has been conducted to evaluate the physiological effects of Hylocereus and Opuntia cacti on endothelial and vascular function in in vivo animal models and human studies. An electronic search was performed in the following databases: PubMed (MEDLINE), EMBASE (via Ovid), CINAHL, Scopus, Web of Science®, and The Cochrane Library (CENTRAL). All journals were searched since inceptions up to January 2020 without language restriction. Outcomes of interest were blood pressure, arterial stiffness, vascular reactivity and biochemical markers of endothelial dysfunction. Two investigators independently performed the study selection and data extraction. From 394 references, only 16 studies ( 9 animal and 7 human) fulfilled the eligibility criteria. Animal studies suggested a potential increase in vasodilation and serum nitric oxide and a reduction in vascular stiffness and blood pressure. The small number of human studies showed a reduction in heart rate as well as an increase in heart rate variability. Although these findings appear to indicate improvement in vascular health, there is a severe lack of robust, randomised human intervention studies to identify underlying mechanisms, optimal dose and long-term effects of cacti consumption.
\end{abstract}

\section{Introduction}

Native to South America and Southeast Asia, the dragon fruit, pitaya (Hylocereus) and the cactus pear, nopal (Opuntia) are edible cacti grown in the tropics for their fruits, skin and cladodes (the pads of the cactus). They are becoming increasingly well-known worldwide in the form of food, snacks and dietary supplements. The popularity of the dragon fruit may initially have been due to its vivid hue and bizarre structure. As for the cactus pear however, it has been used in the Latin America as traditional folk remedies and are harvested today

\footnotetext{
${ }^{a}$ Department of Nutritional Sciences, School of Life Course Sciences, Faculty of Life Science and Medicine, King's College London, London, UK.

E-mail: ana.rodriguez-mateos@kcl.ac.uk

${ }^{b}$ Department of Diabetes, School of Life Course Sciences, Faculty of Life Science and Medicine, King’s College London, London, UK. E-mail: paul.w.caton@kcl.ac.uk $\dagger$ Electronic supplementary information (ESI) available. See DOI: 10.1039/ dofo00537a
}

as a crop vegetable becoming a staple in the modern diet in that region. ${ }^{1}$ Most importantly, they also contain high levels of soluble fibre ${ }^{2,3}$ and bioactive phytochemicals such as betalains, ${ }^{4,5}$ the beneficial properties of which have sparked considerable interests among researchers. Betacyanins and betaxanthins are two subclasses of betalains and are natural colour pigments commonly found in some cacti species and in red beetroot. ${ }^{6}$ Some variants of the cactus pear have been documented to contain up to $8.15 \mathrm{mg} \mathrm{g}^{-1}$ dry fruit of total betalains and is comparable to that of the red beetroot at $8.60 \mathrm{mg} \mathrm{g}^{-1}$ dry tissue which is well-known as a crop grown for their red betalain pigments for use as natural food dyes. ${ }^{7}$ However, betalains content are lower in the dragon fruit as seen in red pitaya (Hylocereus polyrhizus) peel and flesh (3.02 $\mathrm{mg} \mathrm{g}^{-1}$ and $4.27 \mathrm{mg} \mathrm{g}^{-1}$ dry weight respectively, ${ }^{8}$ corrected for $\sim 90 \%$ of moisture content ${ }^{3}$ ). Even though these cacti may also contain some other phytochemicals, the betalains are known to be the most abundant out of their total alkaloidal and phenolic content. In fact, analysis carried out 
on a range of different genotypes of the dragon fruit showed that betalainic compounds occupied an average of $85 \%$ of their total phenolics. ${ }^{9}$ Given that, it is likely that these bioactives could be responsible for their beneficial effects. On the cellular level, indicaxanthin - the most prominent betalain in cactus pear, has been able to strongly inhibit the expression of endothelial intercellular adhesion molecule-1 (ICAM-1) which is a biomarker of atherosclerosis. ${ }^{10}$ Another in vitro experiment reported that indicaxanthin prevents adhesion of human erythrocytes onto the endothelium layer demonstrated on human umbilical vascular endothelial cells (HUVEC). ${ }^{11}$ In animal models, the red pigment betanin ameliorates isoproterenol-induced acute myocardial infarction (AMI) in rats by inhibiting the protein expressions of inducible nitric oxide synthase (iNOS) and nuclear factor-kappa (NF-kB). ${ }^{12}$ The use of pure betalains as treatment for vascular studies in human studies is rather scarce. However, a randomised crossover pilot study investigating betalain-rich red beetroot juice and cooked beetroot on hypertensive individuals reported that two weeks supplementation of red beet juice significantly increased flow-mediated dilation (FMD) while systolic and diastolic blood pressure, ICAM-1, vascular endothelial adhesion molecule-1 (VCAM-1) and E-selectin were reduced markedly. ${ }^{13}$ This is consistent with another human intervention trial involving concentrated red beetroot juice. They also reported a reduction in systolic blood pressure in overweight participants after three weeks of supplementation with the beetroot juice concentrate. ${ }^{14}$ The existing body of research on these cacti are mostly centred around their ameliorative effects on body weight, lipid profile and type- 2 diabetes mellitus. ${ }^{15-17}$ However, research into their benefits on cardiovascular health has gained significant momentum over the last decade. Recent systematic reviews have already reviewed the impact of cactus pear and dragon fruit consumption on body weight, glycaemic control and other cardiovascular risk factors (such as body weight and composition, serum triglycerides, cholesterol and blood glucose). ${ }^{18,19}$ Despite that, their association with endothelial and vascular function has yet to be established. Because the endothelium layer plays the ultimate role of regulating the vascular tone (namely via the endothelial nitric oxide synthase pathway ${ }^{20}$ alongside controlling leukocytes recruitment during endothelial inflammation, ${ }^{21} \mathrm{a}$ disruption in normal endothelial function likely causes endothelial dysfunction - a precursor towards the onset of atherosclerosis $^{22}$ and a useful predictor of cardiovascular events. ${ }^{23} \mathrm{~A}$ systematic review concluded that $1 \%$ increase in FMD decreases cardiovascular risk down to 0.92 (pooled overall risk). ${ }^{24}$ In recent years, techniques used for assessing vascular and arterial function have vastly improved with new technologies. Gold standard non-invasive measurements such as flow-mediated dilation (FMD) and pulse wave velocity (PWV) have been well established for clinical application on patients $^{25,26}$ and further adapted for use in animals. ${ }^{27,28}$ Therefore, the objective of this review was to systematically evaluate available evidence for the effects of the two most common edible cacti on endothelial and vascular function, using data published in animal models and human intervention trials.

\section{Materials and methods}

This systematic review was conducted according to The Cochrane Handbook for Systematic Reviews of Interventions Version 5.1.0 ${ }^{29}$ as well as the Preferred Reporting Items for Systematic Reviews and Meta-Analysis (PRISMA) Guidelines 2009. ${ }^{30}$ A PRISMA checklist is also provided as supplementary document (ESI Document $1 \dagger$ ). This review is registered on PROSPERO (registration No. CRD42017082714).

\section{Search strategy}

A comprehensive search was conducted on six databases, PubMed (MEDLINE), EMBASE \& EMBASE Classic (via Ovid), The Cochrane Library (CENTRAL), Scopus, Cumulative Index of Nursing and Allied Health Literature (CINAHL) and Web of Science ${ }^{\circ}$, searching all years of record up until $15^{\text {th }}$ of January 2020 with no language restriction. All non-English articles were translated by the authors. Reference lists of retrieved articles were also manually searched for relevant publications. A review article evaluating the effects of fruit, vegetable and potassium intake on vascular and endothelial function was used to assist with the development of the concept keywords. ${ }^{31}$ Search terms and MeSH terms were categorised into 2 key concepts: cactus fruits and endothelial or vascular functions. Key search terms were "cactus fruit" OR "Hylocereus" OR "Opuntia” OR "pitaya” OR "nopal” OR "prickly pear" AND "endothelial function*" OR "vascular function*” OR "arterial stiffness" OR "vascular reactivity" OR "blood pressure" OR "nitric oxide" OR "adhesion molecules". An example search query with the complete search terms are provided in Box 1 (ESI Document $2 \dagger$ ).

\section{Eligibility criteria}

Published studies included in this review were required to adhere to the following criteria: (1) original research; (2) animal or human intervention studies investigating the supplemental effects of the fruits, cladodes or extract of the cacti on endothelial and vascular functions. Cactus fruits include Hylocereus and Opuntia species and subspecies. The primary outcomes were defined by results of the following techniques: measures of arterial compliance/stiffness via pulse wave velocity (PWV), pulse wave analysis (PWA), digital volume pulse (DVP), augmentation index (AI), pulse pressure (PP), and Finapres, and vascular reactivity via flow mediated dilation (FMD), laser Doppler imaging (LDI) and peripheral artery tonometry (PAT). Studies with blood pressure (BP), heart rate (HR) and heart rate variability (HRV) as outcome measures were also included. Biochemical markers of endothelial dysfunction included in this review are nitric oxide (NO) production via nitrate/nitrite analysis, ICAM, VCAM, E-selectin and vasoconstrictor peptide endothelin-1 (ET-1). Inclusion criteria in the 
Table 1 Scope of the systematic review in PICOS form

\begin{tabular}{ll}
\hline Criteria & Definition \\
\hline Population & - Animal studies: all animal models (including healthy animals, animals with pre-existing or induced conditions, and animals \\
& subjected to a diet) \\
Interventions & - Human studies: men and women across all ages (including healthy participants, athletes, or participants with disease) \\
& whole fruits, cladodes or extracts of the cacti delivered orally or intravenously \\
Comparator & - Comparison against baseline, placebo group or control groups \\
Outcomes & Endothelial and vascular functions defined by the results of the following techniques: PWV, PWA, DVP, AI, PP, Finapres, FMD, \\
& LDI, and PAT \\
& - Blood pressure (BP) \\
& - Heart rate (HR) and heart rate variability (HRV) \\
Study design & - Animal intervention models \\
& - Human pilot studies and randomised controlled trials
\end{tabular}

form of a PICOS (population, intervention, comparison, outcome, and study design) statement are list in Table 1.

\section{Study selection}

After redundant articles were removed, remaining articles were independently screened by two investigators (AC and TWG) for eligibility based on title and abstract. Eligible articles were put forward for a full-text review when a consensus was reached. Articles with insufficient information in the abstract were also moved to full-text review. In the case of disagreement, a consensus was reached through discussion among the authors (AC, TWG, ARM, and PWC).

\section{Results}

\section{Study selection and characteristics}

Details of literature search and selection of studies are presented in the form a flow chart illustrated in Fig. 1. A total of 394 articles were identified through electronic search but 270 were excluded for being redundant or clearly irrelevant (cacti species not being investigated, ecological or agricultural studies of the cacti, or studies relating to the physical properties of the cacti) on the basis of title and abstract. In the next step, 108 of the 124 potential articles were justifiably excluded after full-text assessment, leaving only 16 papers in total that fulfil the eligibility criteria. Two of the included papers were not in English and were translated: one from Spanish to English ${ }^{32}$ and the other from Simplified Chinese to English $^{33}$ by authors ARM and AC respectively. The characteristics of included animal and human studies are summarised in Tables 2 and 3 respectively. Of the 16 publications, 9 were experiments conducted using animal models while 7 were human studies ( 1 pilot study and 6 randomised controlled trials). Chronic and sub-chronic interventions were described in 10 studies and 6 were acute interventions. Only two studies explored dose-dependence (three to five different doses) and four publications compared only two different doses of cactus fruit administration.

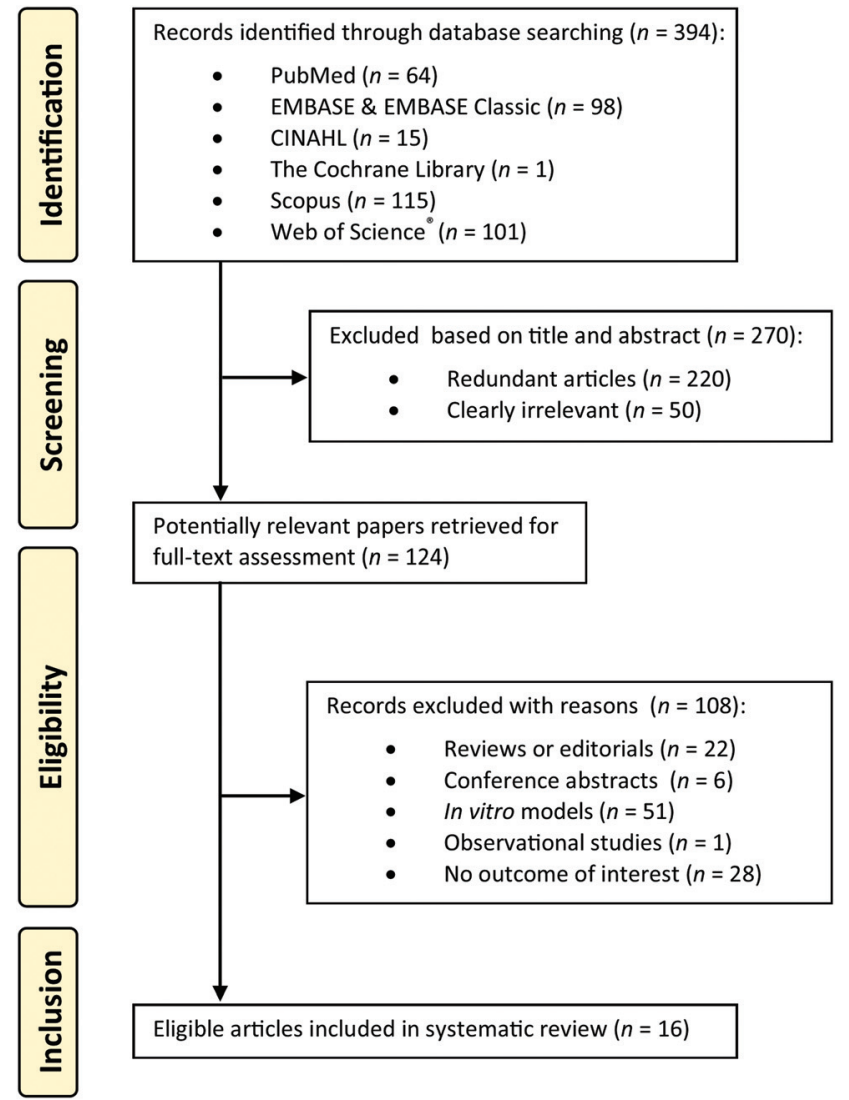

Fig. 1 PRISMA flow diagram for selection of studies.

\section{Animal studies}

Effects of cacti on blood pressure (BP) in animal studies. Among animal studies, five showed significant reductions in $\mathrm{BP}^{34-38}$ In the first study, ${ }^{34} 24$ male Sprague-Dawley rats were randomly divided into four groups: I (control), II (diabetic), III (low dose), and IV (high dose). Group II, III and IV were given Streptozotocin (STZ) injection to induce diabetes. Then only Group III and IV were orally given two different doses (250 and $500 \mathrm{mg}$ per $\mathrm{kg}$ bw respectively) of aqueous extract of white 
Table 2 Characteristics of animal studies investigating the effects of cactus fruits consumption on vascular functions

\begin{tabular}{|c|c|}
\hline Reference & Study details \\
\hline $\begin{array}{l}\text { Anand } \\
\text { Swarup et al. } \\
(2010)^{34}\end{array}$ & $\begin{array}{l}\text { Male Sprague-Dawley rats }(n=24) \text { were } \\
\text { randomly divided into four groups: I (control), } \\
\text { II (diabetic), III (low dose), and IV (high dose). } \\
\text { Group II, III and IV were induced diabetes via } \\
\text { STZ injection and then Group III and IV were } \\
\text { orally administered dragon fruit extract (DFE) } \\
\text { for } 5 \text { weeks. }\end{array}$ \\
\hline $\begin{array}{l}\text { Bakour et al. } \\
(2017)^{35}\end{array}$ & $\begin{array}{l}\text { Normotensive rabbits }(n=8) \text { divided into two } \\
\text { groups: control and treatment. Treatment } \\
\text { group was administered various doses of } \\
\text { lyophilised extract of cladode from cactus pear } \\
\text { via intravenous infusion at different time } \\
\text { points while control group received saline. }\end{array}$ \\
\hline $\begin{array}{l}\text { Cardenas } \\
\text { et al. }(2019)^{36}\end{array}$ & $\begin{array}{l}\text { Male Wistar rats ( } n=21) \text { after three weeks of } \\
\text { high-fructose diet (HFD) were divided into } \\
\text { three groups to receive either mucilage (gel } \\
\text { extraction), fresh nopal cladodes or water } \\
\text { orally for } 30 \text { days. }\end{array}$ \\
\hline
\end{tabular}

Oh and Lim $(2006)^{40}$

Harrat et al. $(2019)^{37}$

Ramli et al. $(2014)^{39}$

Saleem et al. $(2005)^{38}$

Wang et al. $(2015)^{33}$

Zhao et al. $(2012)^{41}$
Male A/J mice $(n=24)$ divided into four groups: control, hyperlipidaemic control and two treatment groups ( 25 and $50 \mathrm{mg} \mathrm{kg}{ }^{-1}$ ). Treatment groups were pre-treated with cactus pear glycoprotein orally for $3 \mathrm{~d}$ before Triton WR-1339 administration.

Male Wistar rats $(n=12)$ were divided into two groups and were subjected to high-fat diet (HFD) for 10 weeks before being rendered diabetic via STZ injection. One group was supplemented with freeze-dried cactus pear powder while the other received none for 30 days.

Male Wistar rats $(n=48)$ randomly divided into four groups: corn-starch (CS), corn-starch + red pitaya juice (CRP), high-carbohydrate, high fat group (HCHF) and high carbohydrate, high fat + red pitaya juice (HRP). All groups were subjected to the corresponding diet for 8 weeks before supplemented with red pitaya for another 8 weeks.

Two groups of Sprague-Dawley rats (10 rats per group) were administered two different doses of methanolic extract of spiny pest pear via intravenous injection.

Male Sprague-Dawley rats $(n=24)$ randomly divided into four groups: control, atherosclerotic control, low dose treatment and high dose treatment group. Atherosclerotic control and treatment groups were subjected to 30 days of high-fat and highcalcium diet before administration of spiny pest pear polysaccharide (OPS) via intraperitoneal injection for $60 \mathrm{~d}$.

Male Sprague-Dawley rats $(n=60)$ randomly divided into six groups: I (control), II (hyperlipidaemic control - high-fat emulsion), III (positive control - high-fat emulsion + Zhibituo tablet), IV-VI (treatment group high-fat emulsion $+100,200$, and $400 \mathrm{mg}$ per $\mathrm{kg}$ bw of ODP-Ia respectively). Treatments were administered orally for $28 \mathrm{~d}$.

Treatment

Aqueous extract; from white pitaya pulp (Hylocereus undatus)

250 and $500 \mathrm{mg}$ per $\mathrm{kg}$ bw; for $35 \mathrm{~d}$ (5 weeks)

Lyophilised extract of cladode power; from cactus pear (Opuntia ficus-indica)

$40,60,80$ and $100 \mathrm{mg}$ per $\mathrm{kg}$ bw at $0,20,40$, and $60 \mathrm{~min}$ respectively Group 1: mucilage of cladodes; of cactus pear (Opuntia ficus-indica) Group 2: liquefied nopal cladodes; of cactus pear (Opuntia ficus-indica) $500 \mathrm{mg}$ per $\mathrm{kg}$ bw and $4.36 \mathrm{~g}$ per $\mathrm{kg}$ bw respectively; for 30 d

Glycoprotein (90 kDa); from cactus pear (Opuntia ficusindica)

25 and $50 \mathrm{mg}$ per $\mathrm{kg} \mathrm{bw}$; for $3 \mathrm{~d}$

Freeze-dried powder; of cactus pear (Opuntia ficusindica)

$5 \%$ of total diet; for $30 \mathrm{~d}$

Juice; of red pitaya (Hylocereus polyrhizus) $5 \%$ red pitaya juice in diet; for $56 \mathrm{~d}$ (8 weeks)

Methanolic extract; of cladodes of spiny pest pear (Opuntia dillenii)

1 and $10 \mathrm{mg}$ per $\mathrm{kg}$ bw Polysaccharide; of spiny pest pear (Opuntia dillenii) Low: $0.2 \mathrm{~g} \mathrm{~kg}^{-1} \mathrm{~d}^{-1}$ High: $0.4 \mathrm{~g} \mathrm{~kg}^{-1} \mathrm{~d}^{-1}$; for 60 days

ODP-Ia from aqueous extract; of spiny pest pear (Opuntia dillenii) 100, 200, and $400 \mathrm{mg}$ per $\mathrm{kg}$ bw; for $28 \mathrm{~d}$
Results

$\mathrm{SAP} \downarrow$ following supplementation (250 mg per $\mathrm{kg}$ bw) compared to diabetic control group $(P$ $<0.05$ ).

No difference on DAP and MAP observed. $\mathrm{PWV} \downarrow$ and PP $\downarrow$ significantly in both treatment groups $v s$. control $(P<0.05)$.

MAP $\downarrow$ in a dose-dependent manner following extract administration compared to control group $(P<0.05)$

No difference in SBP compared to control group.

$\mathrm{DBP} \downarrow$ significantly only in fresh nopal group compared to control group $(P<0.05)$.

NO $\uparrow$ significantly in plasma of treatment group (50 $\mathrm{mg}$ per $\mathrm{kg}$ bw) compared to hyperlipidaemic control group $(P<0.05)$.

SBP $\downarrow$ and DBP $\downarrow$ significantly in treatment group compared to control group $(P<0.001)$.

Diastolic stiffness $\downarrow$ (ex vivo) significantly following supplementation in corn-starch + red pitaya group (CRP) compared to cornstarch control $(\mathrm{CS})(P<0.05)$.

MABP $\downarrow$ significantly by $27.65 \%$ and $54.12 \%$ after administration of spiny pest pear extract at 1 and $10 \mathrm{mg}$ per $\mathrm{kg}$ bw respectively $(P<$ $0.001)$.

Vasorelaxation $\uparrow$ (thoracic aorta) following administration of treatment in both low and high dose treatment group compared to atherosclerotic control group $(P<0.01)$.

Serum NO $\uparrow$ following treatment administration in medium and high dose treatment group (200 and $400 \mathrm{mg}$ per $\mathrm{kg}$ bw) compared to hyperlipidaemic control group $(P$ $<0.01)$.

bw, body weight; DAP, diastolic arterial pressure; MABP, mean arterial blood pressure; MAP, mean arterial pressure; NO, nitric oxide; PP, pulse pressure; PWV, pulse wave velocity; SAP, systolic arterial pressure; STZ, streptozotocin. 
Table 3 Characteristics of human studies investigating the effects of cactus fruits consumption on vascular functions

\begin{tabular}{|c|c|c|c|}
\hline $\begin{array}{l}\text { Fabela-Illescas } \\
\text { et al. }(2015)^{32}\end{array}$ & $\begin{array}{l}\text { Pilot study with no control. } 20 \text { Individuals } \\
(10 \text { with T2DM and the rest with } \\
\text { hypertension, overweight and obesity) were } \\
\text { fasted every morning and supplemented with } \\
\text { prickly pear juice drink for } 30 \text { days. Vascular } \\
\text { measurements were taken at baseline, } 8 \mathrm{~d}, 15\end{array}$ & $\begin{array}{l}\text { Juice drink; of prickly pear } \\
\text { cladodes (Nopalea cochenilliferal } \\
\text { Opuntia cochenillifera) } \\
\text { Juice containing } 50 \mathrm{~g} \text { of prickly } \\
\text { pear cladodes }+250 \mathrm{~mL} \text { water; } \\
\text { for } 30 \mathrm{~d}\end{array}$ & $\begin{array}{l}\text { DBP } \downarrow \text { significantly at } 23 \mathrm{~d} \text { post- } \\
\text { supplementation compared to baseline }(P< \\
0.05) \text {. } \\
\text { SBP } \downarrow \text { significantly at } 15 \mathrm{~d}, 23 \mathrm{~d} \text {, and } 30 \mathrm{~d} \\
\text { post-supplementation compared to baseline } \\
(P<0.05) .\end{array}$ \\
\hline
\end{tabular}

Grube et al. $(2013)^{16}$ $\mathrm{d}, 23 \mathrm{~d}$ and $30 \mathrm{~d}$.

$(2013)^{16}$
controlled trial. 123 individual (30 male and
$33-57.1$ year were advised on physical activity
and hypocaloric diet plans. They were then
randomly assigned to receive either $3 \mathrm{~g}$ day
of litramine tablets or a placebo after a
2-week placebo run-in phase.

Khouloud Randomised, controlled trial. 22 male et al. $(2018)^{42}$ athletes (20-22 years) randomly assigned to receive daily $150 \mathrm{~mL}$ of prickly pear juice or no intervention at all for 2 weeks. Participants were subjected to yo-yo intermittent recovery test before and after intervention.

Rahimi et al. Randomised, double-blind, placebo$(2019)^{43} \quad$ controlled, crossover trial. 48 non-smoking men (45-55 years, BMI: $25-35 \mathrm{~kg} \mathrm{~m}^{-1}$ ) with coronary artery disease $(\mathrm{CAD})$ all received the placebo, betalain-rich extract of red beetroot, and betacyanin-rich extract of cactus pear fruit on three occasions separated by two weeks wash out.

Schmitt et al. Randomised, placebo-controlled, crossover $(2008)^{45} \quad$ trial. 10 male professional skiers aged $23.8 \pm$ 4.5 years were randomly assign to receive either the OFI (Opuntia ficus-indica) capsule or the placebo at the end of the training day ( $3 \mathrm{~h}$ after controlled meal). Crossover protocol was applied after a month of washout period.

Van Proeyen Randomised, double-blind, placeboet al. $(2012)^{46} \quad$ controlled, crossover trial. Physically active men $(n=6)$ aged $21.0 \pm 1.6$ years were fasted overnight and randomly assigned to receive either OFI extract capsule or the placebo followed by a series of cycling exercise. Crossover protocol was applied after 2-week washout period.

Wiese et al. Randomised, double-blind, placebo$(2004)^{44}$ controlled, crossover trial. Healthy individuals $(n=55,23$ male and 32 female) aged 21-35 years were randomly assigned to receive either 1600 IU of cactus pear extract or placebo $5 \mathrm{~h}$ before alcohol consumption.
Litramine tablets (natural fibre complex); derived from cactus pear (Opuntia ficus-indica)

$2 \times 3 \times 500 \mathrm{mg}$ ( $3 \mathrm{~g}$ total $)$ IQP tablets per day; for $56 \mathrm{~d}(12$ weeks)

Juice drink; of prickly pears (Opuntia ficus-indica) $3 \times 50 \mathrm{~mL}$ juice per day; for 14 d (2 weeks)

Betacyanin-rich extract capsule; of cactus pear (Opuntia stricta) containing $25 \mathrm{mg}$ betacyanins. 2 capsules (50 mg total) per day; for $14 \mathrm{~d}$ (2 weeks)

Extract capsule; of prickly pears (Opuntia ficus-indica) 1 capsule (equiv. to $100 \mathrm{~g}$ fresh fruit) per $20 \mathrm{~kg} \mathrm{bw}$

Extract capsule (OpunDia); of prickly pears (Opuntia ficusindica)

$6 \times 333.3 \mathrm{mg}$ capsule $(2000 \mathrm{mg}$ total)

Extract capsule; of cactus pear (Opuntia ficus-indica) $2 \times 800$ IU capsule
No significant difference was observed for SBP, DBP and HR following supplementation in treatment group compared to placebo group.

No significant difference was observed for SBP, DBP following supplementation. $\mathrm{HR} \downarrow$ significantly in treatment group versus control $(P<0.05)$.

SBP $\downarrow$ significantly after consumption of cactus pear capsule $(P<0.0001)$.

No significant difference in DBP was reported.

$\mathrm{HRV} \uparrow\left(\mathrm{HF}_{\mathrm{SU}}, \mathrm{LF}_{\mathrm{SU}}, \mathrm{HF}_{\mathrm{ST}}, \mathrm{LF}_{\mathrm{ST}} \uparrow\right)$ significantly in OFI group compared to control group. HR $\downarrow$ significantly in OFI group versus control group.

HR was consistently lower in OFI (by $~ 10$ beats per min) throughout exercise compared to control group.

No significant difference was observed for SBP, DBP and HR following supplementation in treatment group compared to placebo group.

$\mathrm{BP}$, blood pressure; DBP, diastolic blood pressure; HR, heart rate; $\mathrm{HF}_{\mathrm{ST}}$, high frequency (standing); HF $\mathrm{SU}_{\mathrm{SU}}$, high frequency (supine); HRV, heart rate variability; $\mathrm{SBP}$, systolic blood pressure; $\mathrm{LF}_{\mathrm{ST}}$; low frequency (standing); $\mathrm{LF}_{\mathrm{SU}}$, low frequency (supine); $\mathrm{T} 2 \mathrm{DM}$, type-2 diabetes mellitus.

dragon fruit (Hylocereus undatus) pulp for 35 days. After the intervention, systolic arterial pressure (SAP) in Group III has returned to baseline level and is significantly lower compared to diabetic group (Group II) (124.2 \pm 2.1 vs. $130.2 \pm 1.3 \mathrm{mmHg}$, $P<0.05)$ and surprisingly, the group with the higher dose (Group IV) showed no changes. However, there were also no difference in diastolic arterial pressure (DAP) and mean arterial pressure (MAP) across all groups. On another study, ${ }^{36}$ male Wistar rats $(n=21)$ were subjected to three weeks of high-fruc- tose diet and then randomly divided into three groups: (1) control group; (2) group receiving $500 \mathrm{mg}$ per $\mathrm{kg}$ bw mucilage of cactus pear cladodes; (3) group receiving $4.36 \mathrm{~g}$ per $\mathrm{kg}$ bw fresh liquefied cactus pear cladodes. After 30 days of treatment, they observed a significant decrease in diastolic blood pressure (DBP) in the group that had the fresh cactus pear (Opuntia ficus-indica) when compared to control $(P<0.05)$ although the absolute change was not reported by the authors. Systolic blood pressure (SBP) however did not change at all for 
any treatment group. Harrat et al. reported similar finding in their study. ${ }^{37}$ Briefly, male Wistar rats $(n=12)$ were divided into two groups and were put on a high-fat diet for 10 weeks before being rendered diabetic via STZ. One group was supplemented with $5 \%$ (of total diet weight) of freeze-dried cactus pear powder on top of their ongoing high-fat diet while the other group received no treatment for a duration of 30 days. When compared to control group, both SBP and DBP substantially decreased by $16 \%(107.6 \pm 3.5$ vs. $127.9 \pm 7.3 \mathrm{mmHg}, P<$ $0.001)$ and $20 \%(70.4 \pm 2.1$ vs. $87.9 \pm 6.2 \mathrm{mmHg}, P<0.001)$ respectively.

The other two studies were acute studies and both administered the intervention via intravenous injection. They both have reported a decrease in MAP. In Bakour et al., ${ }^{35}$ normotensive rabbits $(n=8)$ were divided into two groups: control and treatment. Treatment group was intravenously administered increasing dose of lyophilised extract of cactus pear cladodes in $20 \mathrm{~min}$ intervals over one hour $(40,60,80$ and $100 \mathrm{mg}$ per $\mathrm{kg}$ bw at $0,20,40$, and 60 min respectively). ${ }^{35}$ MAP dropped sharply in a dose-response manner at $20 \mathrm{~min}$ onwards (Control: $103.25 \pm 2.16,98.75 \pm 1.24,101.50 \pm 2.19,101.25 \pm$ $1.13 \mathrm{mmHg}$ vs. Treatment: $80.84 \pm 4.16,58.10 \pm 1.85,50.60 \pm$ $1.57,45.17 \pm 1.52 \mathrm{mmHg}, P<0.05)$. The other acute study also utilised a similar study design with larger sample size, but without a control group. In this study, ${ }^{38}$ normotensive Sprague-Dawley rats in two groups (10 rats per group) were anaesthetised and were each given one-off treatment of 1 or $10 \mathrm{mg}$ per $\mathrm{kg}$ bw of methanolic extract of spiny pest pear (Opuntia dillenii) via intravenous injection. After the treatment, MAP significantly decreased by $27.65 \%$ for the low dose $(119.60 \pm 3.75$ vs. $86.53 \pm 8.47 \mathrm{mmHg}, P<0.001)$ and $54.12 \%$ for the high dose $(123.27 \pm 4.62$ vs. $56.56 \pm 6.04 \mathrm{mmHg}, P<$ 0.001 ) from baseline values.

Effects of cacti on vascular stiffness, vascular reactivity and other biomarkers in animal studies. Data on vascular stiffness, vascular reactivity and vascular biomarkers were only reported in five animal studies. The group who reported BP improvement in diabetic rats $^{34}$ after supplementation of white dragon fruit extract also reported significant reduction in PWV and PP. After the intervention period, PWV of Group II (diabetic) was significantly greater than Group I (control) (6.54 \pm 0.12 vs. $\left.5.46 \pm 0.09 \mathrm{~m} \mathrm{~s}^{-1}, P<0.05\right)$ and with supplementation of the extract the PWV significantly decreased $(P<0.05)$ in both treatment group (Group III and IV) but author did not provide the absolute values to these changes. They also reported a significant reduction in PP for both treatment groups compared to the diabetic group (Diabetic: $40.2 \pm 0.9$ vs. Group III: $37.3 \pm 0.8$ and Group IV: $37.3 \pm 1.0 \mathrm{mmHg}, P<0.05)$. Similar effects were also observed in a recent study. ${ }^{39}$ There, 48 male Wistar rats were divided into four groups: corn-starch (CS), corn-starch + red pitaya juice (CRP), high-carbohydrate, high fat group (HCHF) and high carbohydrate, high fat + red pitaya juice (HRP). All groups followed their designated diet for 56 days and then two of the four were supplemented with $5 \%$ red dragon fruit pitaya (Hylocereus polyrhizus) juice for another 56 days making a total of 112 days. Across all groups, only rats with the normal corn-starch + red pitaya (CRP) diet showed significantly lower diastolic stiffness $(P<0.05)$ compared to cornstarch (CS) control but the absolute values were not presented.

In another approach, ${ }^{33}$ Sprague-Dawley rats $(n=24)$ were divided into four groups: control, atherosclerotic group, low dose and high dose. All groups except the control were subjected to 30 days of high fat, high calcium diet. Thereafter, the low dose and high dose group were intravenously administered a low and high dose ( 0.2 and $0.4 \mathrm{~g}$ per $\mathrm{kg}$ bw for 60 days) respectively of polysaccharide $(77.3 \%$ purity) derived from spiny pest pear. After the diet regime, the atherosclerotic model group showed marked reduction in vasorelaxation ratio induced by carbamylcholine chloride (Carb) at $5 \mu \mathrm{mol} \mathrm{L} \mathrm{L}^{-1}$ versus the control $(0.14 \pm 0.04$ vs. $0.98 \pm 0.02, P<0.01)$. The low dose treatment group has significantly regained vasorelaxation compared to the atherosclerotic model $(0.34 \pm 0.08$ vs. $0.14 \pm$ $0.04, P<0.01)$. The group received the higher dose demonstrate even greater improvement $(0.98 \pm 0.02$ vs. $0.14 \pm 0.04, P<$ 0.01 ) and the values were on par with the control.

A total of two studies explored effects on molecular biomarkers. In the first study, ${ }^{40} 24$ male $\mathrm{A} / \mathrm{J}$ mice were split into 4 groups: control, hyperlipidaemic, low dose, high dose group. Both treatment group orally received different doses (25 or $50 \mathrm{mg}$ per $\mathrm{kg}$ bw for 3 days) of glycoproteins (95\% purity, $90 \mathrm{kDa}$ ) from cactus pear. After 3-days of pre-treatment, all groups except the control were induced hyperlipidaemia with Triton WR-1339 via injection. The serum NO in the hyperlipidaemic group reduced significantly $(P<0.05)$ versus control. Only mice which received the higher dose showed marked increase in serum NO (by $10.4 \mu \mathrm{M})(P<0.05)$ compared to the hyperlipaedemic model. Absolute values for each group were not reported however. Consistent observations were also reported by a more recent attempt with hyperlipidaemic rat models. ${ }^{41}$ In the study, Male Sprague-Dawley rats ( $n=60,10$ per group) were randomly divided into six groups: I (control), II (hyperlipidaemic control highfat emulsion), III (positive control - high-fat emulsion + positive control drug, Zhibituo tablet), IV-VI (treatment group high-fat emulsion $+100,200$, and $400 \mathrm{mg}$ per $\mathrm{kg}$ bw of ODP-Ia respectively). Their treatment (ODP-Ia) is an aqueous extract of polysaccharide $(98.62 \%$ purity) from the spiny pest pear. Treatments were orally administered once daily for 28 days. At the end of the intervention, the hyperlipidaemic control group showed significantly lower serum NO compared to the normal control and positive control group $(10.4 \pm 2.02$ vs. $20.8 \pm 1.49$ and $19.3 \pm 1.19 \mathrm{mmol} \mathrm{L}^{-1}$ respectively, $\left.P<0.01\right)$. With the treatment, the serum NO increased dose-dependently across all three doses but only the medium dose $(14.5 \pm 1.70$ vs. $10.4 \pm$ $\left.2.02 \mathrm{mmol} \mathrm{L}^{-1}, P<0.01\right)$ and high dose $(19.2 \pm 1.65$ vs. $10.4 \pm$ $\left.2.02 \mathrm{mmol} \mathrm{L}^{-1}, P<0.01\right)$ demonstrated significant improvement against the hyperlipidaemic control group.

\section{Human studies}

Effects of cacti on blood pressure (BP) in human studies. In terms of clinical evidence, a total of five human studies ${ }^{16,32,42-44}$ explored changes in BP but only two ${ }^{32,43}$ reported significant improvements. The first study is a pilot 
study with no placebo group to compare against the treatment group. ${ }^{32}$ Twenty individuals with cardiometabolic disorders (10 with type-2 diabetes and the rest with hypertension, overweight and obesity) were fasted and then given $250 \mathrm{~mL}$ of diluted cactus pear juice (containing $50 \mathrm{~g}$ of prickly pear cladodes) for 30 days. SBP significantly lowered from the $15^{\text {th }}$ day onwards, while the decrease in DBP was only significant on the $23^{\text {rd }}$ day post-supplementation. However, the absolute change (in $\mathrm{mmHg}$ ) or magnitude of change was not reported by the authors. In the most recent crossover RCT conducted on non-smoking men ( $n=48$, aged 45-55, BMI: $\left.25-35 \mathrm{~kg} \mathrm{~m}^{-1}\right)$ with coronary artery disease (CAD), participants who were given daily capsules of betacyanin-rich extract (containing $50 \mathrm{mg}$ of total betacyanin) of cactus pear for two weeks showed significant reduction in SBP of $3.16 \mathrm{mmHg} .{ }^{43}$ Contradictory findings were reported in the other three RCTs. ${ }^{16,42,44}$ Grube et al. found no changes in SBP and DBP when overweight and obese individuals $(n=123,30$ male and 93 female, aged 33-57) consumed $3 \mathrm{~g}^{-1 a y}{ }^{-1}$ of litramine tablets (fibre complex from cactus pear) or placebo for a total of 56 days. ${ }^{16}$ Similarly, Wiese et al. showed in their acute RCT crossover trial that cactus pear extract (1600 IU) did not significantly reduce SBP and DBP in healthy individuals $(n=55,23$ male and 32 female, aged 21-35). ${ }^{44}$ Lastly in an exercise trial, ${ }^{42}$ SBP and DBP also did not change in male athletes $(n=22$, aged 20-22) after consuming $150 \mathrm{~mL}$ of prickly pear juice daily for two weeks before they were being tested with the yo-yo intermittent recovery exercise test.

Effects of cacti on heart rate (HR) and heart rate variability (HRV) in human studies. The cacti's effect on HR and HRV were only investigated in human RCTs. ${ }^{16,42,44-46}$ Besides the lack of significant impact on BP, the same RCTs conducted by Grube et al. and Wiese et al. also showed no changes in HR. ${ }^{16,44}$ In contrast, data presented in three sports and exercise RCTs suggested otherwise. ${ }^{42,45,46}$ Firstly, a crossover RCT concluded that prickly pear was able to significantly lower HR ( -9 beats per min) and simultaneously increase HRV in both high (HF) and low frequency (LF) components of both supine (SU) and standing (ST) positions. ${ }^{45}$ These effects were evident after male professional skiers $(n=10$, aged $23.8 \pm 4.5)$ consumed prickly pear extract (1 capsule per $20 \mathrm{~kg}$ bw; equiv. to $100 \mathrm{~g}$ fresh fruit) at the end of their training. The other RCT, also a crossover study, yielded similar results on $\mathrm{HR}^{46}$ In physically active men $(n=6$, aged $21.0 \pm 1.6)$, HR was consistently lower by $\sim 10$ beats per min versus placebo group after consuming $2000 \mathrm{mg}$ of prickly pear extract (OpunDia) before a series of cycling exercise. Furthermore, similar trend was also seen in the trial with the recovery exercise test. ${ }^{42}$ The maximum HR during exercise was found to be significantly lower in the treatment group ( -3.64 beats per min) versus control.

\section{Discussion}

Diets rich in phytochemicals and dietary fibre have been shown to have a positive impact on cardiovascular health. This systematic review of animal and human studies provides evidence that supplementation of edible betalain-rich cacti Hylocereus and Opuntia may have potential to influence endothelial and vascular function.

\section{Animal studies}

Interventions that administered very high doses (400-500 mg per $\mathrm{kg}$ bw) of cacti extract in the animal studies failed to show much more of an effect when compared to studies using physiologically and dietary realistic amounts (1-10 mg per $\mathrm{kg}$ $\mathrm{bw}$ ). This dose-response has also been reported elsewhere both in animal and human models where the medium or low dose was shown to be more effective. ${ }^{15,47}$ A possible explanation for this might be that the responsible bioactive components in the cacti or extract may exert synergistic interactions at lower amounts or perhaps contribute to a threshold effect. Such interactions between bioactives have been demonstrated by Rodriguez-Mateos et al. between dietary polyphenols and nitrate ${ }^{48}$ In this review, two studies that explored dosedependence reported a dose-dependent relationship. ${ }^{35,41}$

Although promising, the evidence is limited and it is important to point out the various issues surrounding the findings. In three of the animal studies, the treatments were administered to the animals intravenously via injection or infusion. ${ }^{33,35,38}$ The results they obtained on the change of BP were very dramatic (up to $-66.7 \mathrm{mmHg}$ ). ${ }^{38}$ From a nutritional standpoint, these findings become highly questionable on the basis that the treatments have completely bypassed the gastrointestinal (GI) tract and the GI tract is well characterised as the primary site of absorption and metabolism for many nutrients and food bioactives. In a recent pharmacokinetic study of betalains (the major phytochemicals in beetroot and cacti species), Sawicki et al. detected numerous betalains metabolites in plasma and urine of human volunteers after consumption of red beet juice. ${ }^{49}$ This further highlights the importance of the GI tract when the absorption and transformation of cacti betalains are concerned. Especially because only very little is known about the metabolism of betalains via gut microbiome at this stage. Another limitation to take note of regarding two of the animal studies is that the outcome measures (arterial stiffness and vasorelaxation) were assessed ex vivo, whilst the methodology used for analysis were not the most optimal for those outcomes. ${ }^{33,39}$ Because the regulation of vascular tone and arterial stiffness involve complex cellular, molecular and neurogenic mechanisms, ${ }^{50}$ evaluating them ex vivo raises doubts and will negatively impact the extrapolability of the findings. Ideally, measurements for arterial stiffness and vascular reactivity should be done non-invasively on living subjects using carotid-femoral pulse wave velocity (the gold standard) and flow-mediated dilation (FMD) respectively. The use of such methods in animals have already been well documented in recent studies. ${ }^{27,28}$ Besides the technical issues, the study animals used were also not representative of a healthy population. Almost all of the animal studies reviewed have used diseased animals or animals subjected to high-fructose or high-fat diet as opposed to healthy subjects. Because some 
of the results may seem quite dramatic, this begs the question of whether the treatment would generate similar response on a healthier population. As a whole, evidence from animal studies is reasonably weak from a nutritional and methodological perspective as almost half of the studies suffered from obvious flaws and lack robustness.

\section{Human studies}

On the other hand, results gathered from the human studies are slightly more accountable given the fact that the majority of them are RCTs which is the gold standard for clinical research. Besides, four out of five of the RCTs incorporated a crossover protocol as opposed to a parallel design in an effort to reduce intraparticipant variability. ${ }^{44-46}$ Regardless of that, there is plenty of room for improvement for future approaches. In particular, more adequate placebos (micro and macronutrient matched, identical in taste and appearance to the intervention) are much needed in RCTs and it is necessary to quantify the composition of foods or treatment given to participants, especially on the components that may potentially exert vascular effects like dietary nitrate, fibre and their respective phytochemicals such as betalains and phenolic acids in the case of edible cacti. This is one of the biggest limitations affecting both animal and human studies. Lastly, further insights into the dose and time response as well as pharmacokinetics and pharmacodynamics of betalains and other potential bioactive components in cacti are also strongly encouraged. This will help understand the bioavailability and metabolism of the responsible phytochemicals.

The positive impact of cacti on BP was observed in two studies lasting 2 to 4 weeks only in population with CAD and Type-2 diabetes mellitus (T2DM) of a generally young age range (25-55 years). ${ }^{32,43}$ This warrants future approaches to investigate the effects on healthier populations of a wider age group over a longer period. Although no controls were implemented in one of the studies, ${ }^{32}$ the dosage used was the most nutritionally realistic among all of the studies. Their treatment was a $250 \mathrm{~mL}$ diluted prickly pear juice drink containing only $50 \mathrm{~g}$ of the cactus cladodes. This is easily achievable in the daily diet. In fact, this is even smaller than one 5-A-Day UK portion size $(80 \mathrm{~g})$ for fruits and vegetables. Even though the difference in SBP and DBP was significant $(p<$ 0.05), no additional information was available regarding the absolute change of BP in mmHg. In line with the previous study, the RCT conducted on men with CAD reported significant reduction of SBP $(3.16 \mathrm{mmHg}, p<0.0001)$ with only two weeks of intervention with betacyanin-rich extract derived from cactus pear. ${ }^{43}$ Unlike the other studies, the intervention capsules given to the participants contained only betacyanins (25 mg per capsule). This strongly implicates that the improvement in SBP could be attributed to betalains. This is also consistent with another RCT (not included in this review) investigating the acute effects of beetroot-enriched bread on $24 \mathrm{~h}$ ambulatory BP in healthy male individuals. ${ }^{51}$ When comparing between control bread (CB) vs. white beetroot bread (WBB) vs. red beetroot bread (RBB), RBB was more effective at lowering
DBP $(p<0.05)$ across $0-24 \mathrm{~h}$ post-consumption. Essentially, the WBB and RBB were both matched for all macro and micronutrients including dietary fibre and nitrate (which is also a strong vasoactive agent), ${ }^{52}$ leaving only betacyanins as the sole difference between the two. This further reinforces the notion that these bioactive betalains may play an important role in the cardioprotective effects of these edible cacti. As for HR and HRV, reasonably strong evidence was presented in two robustly designed acute crossover RCT. The study populations were young (21-24 years) physically active men or professional athletes. Because these were sports and performance studies and HR will inevitably increase with aerobic exercise, HR were compared between groups instead. Van Proeyen et al. documented a 10 beats per min lower HR after consuming the prickly pear extract (OpunDia) in treatment group but the composition or fresh fruit equivalent of the extract were not provided. ${ }^{46}$ These results are almost identical to that of Schmitt et al. ${ }^{45}$ In their study, the treatment group also has 9 beats per min lower HR after consuming similar prickly pear extract (equiv. to $300 \mathrm{~g}$ fresh fruit per adult). This dosage may seem quite high but is still well within realistically achievable amounts. In addition to HR, HRV was also higher in treatment groups. In Khouloud et al., their sub-chronic exercise trial yielded similar improvement after two weeks of intervention. ${ }^{42}$ Despite a longer intervention, the reduction in maximum $\mathrm{HR}$ ( -3.64 beats per min) was smaller compared to the RCTs, likely because the amount administered was lower and more realistic to achieve as part of a normal diet ( $150 \mathrm{~mL}$ day $^{-1}$ cactus pear juice). Collectively, HR and HRV are strong biomarkers for predicting stroke and coronary heart diseases. Hence, it is fair to suggest that cacti supplementation may potentially help ameliorate such conditions although much research is still needed to confirm this.

On the opposite end, Grube et al. reported no changes in HR, SBP and DBP in volunteers following supplementation of $3 \mathrm{~g}$ day $^{-1}$ Litramine tablets. ${ }^{16}$ The authors claimed that the Litramine tablet (IQP G-002AS) is a proprietary natural fibre complex derived from the cactus pear (Opuntia ficus-indica) to be used as a weight loss therapy. Because of that, it seems plausible that their observations on vascular parameters were attributed to the lack of the cacti's native vasoactive phytochemicals in their treatment. Likewise, this was also reported in the other RCT looking at the effects of cactus pear on alcohol hangover. ${ }^{44}$ The study also found no difference in HR, SBP and DBP between the treatment and control group. Although this time the complete extract of cactus pear was used, it is important to take note that the vascular measurements were not the primary outcome. Their main objective, however, is to assess the effects of cactus pear on hangover recovery hence the participants were subjected to moderately high levels of alcohol. Due to the fact that dietary alcohol is a strong diuretic and vasoactive agent, it may be possible that the vascular effects in this case were masked by the effects of alcohol consumption.

Overall, all five animal studies exploring effects on BP reported significant reductions, one reported improvement in vascular tone, two observed elevated serum nitric oxide 
and two reported a decrease in arterial stiffness. As for the human studies, only two out of five found reductions in BP, three out of five reported lower heart rate, and the only study investigating the effects on HRV reported a significant increase.

Strengths and limitations. This review has several notable strengths; it involved a comprehensive approach to systematically identify relevant studies without any language or date restriction, and included data from all eligible studies regardless of the experimental designs. Furthermore, two reviewers independently reviewed the titles and abstracts identified. However, the main limitation of this review is the lack of quantitative analysis. Due to high heterogeneity and small amount of studies available, it was concluded from the qualitative review that a conclusive summary estimate may not be possible through meta-analysis. The method of administration is vastly different (oral, injection, infusion). There is also a huge variation in the duration of studies (1-60 days), composition of intervention (juice, extract, glycoprotein, polysaccharide and natural fibre), dose (1-500 mg per kg bw per day), study population (diseased, healthy and athletic), study design (exercise trials as opposed to vascular studies), baseline status and reporting of outcome measures (e.g., absolute change of BP in $\mathrm{mmHg}$, or change in percentage).

\section{Conclusions}

In conclusion, the findings presented in this systematic review suggests that supplementation of the edible cacti Hylocereus and Opuntia appears to improve vascular and endothelial function despite the evidence on BP is mixed. Although the results from animal studies are less promising, the human intervention trials provided evidence that these cacti improves heart rate and increase heart rate variability in both healthy and in population at risk of CVD. Taken together, this may implicate a potential use of these cacti as a novel therapeutic option to remedy vascular dysfunction and other cardiovascular-related disorders. The current review also highlighted a few important gaps. Particularly, the longterm effects of cacti supplementation still remain unclear. Moreover, the optimal amount for human consumption is still unknown due to the lack of dose-dependence design in the RCTs. Because the study population were generally young, there remains a need to explore the effect of cacti supplementation in older populations. Therefore, more robustly designed RCTs are much warranted, with a particular focus on determining both the acute and chronic effects across wider age groups. In addition, attention to exploring the dose-response reaction will help in identifying the optimal dose to inform future research.

\section{Conflicts of interest}

There are no conflicts to declare.

\section{References}

1 M. P. Griffith, The origins of an important cactus crop, Opuntia ficus-indica (Cactaceae): new molecular evidence, Am. J. Bot., 2004, 91, 1915-1921.

2 M. Ennouri, I. Ammar, B. Khemakhem and H. Attia, Chemical Composition and Antibacterial Activity of Opuntia Ficus-Indica F. Inermis (Cactus Pear) Flowers, J. Med. Food, 2014, 17, 908-914.

3 M. C. Jerônimo, J. V. C. Orsine, K. K. Borges and M. R. C. G. Novaes, Chemical and Physical-Chemical Properties, Antioxidant Activity and Fatty Acids Profile of Red Pitaya [Hylocereus Undatus (Haw.) Britton \& Rose] Grown In Brazil, J. Drug Metab. Toxicol., 2015, 6, 1-6.

4 J. C. Guevara, P. Suassuna and P. Felker, Opuntia Forage Production Systems: Status and Prospects for Rangeland Application, Rangel. Ecol. Manag., 2009, 62, 428434.

5 D. H. Suh, S. Lee, D. Y. Heo, Y.-S. Kim, S. K. Cho, S. Lee and C. H. Lee, Metabolite Profiling of Red and White Pitayas (Hylocereus polyrhizus and Hylocereus undatus) for Comparing Betalain Biosynthesis and Antioxidant Activity, J. Agric. Food Chem., 2014, 62, 8764-8771.

6 P. Rahimi, S. Abedimanesh, S. A. Mesbah-Namin and A. Ostadrahimi, Betalains, the nature-inspired pigments, in health and diseases, CRC Crit. Rev. Food Technol., 2019, 59, 2949-2978.

7 E. Castellanos-Santiago and E. M. Yahia, Identification and Quantification of Betalains from the Fruits of 10 Mexican Prickly Pear Cultivars by High-Performance Liquid Chromatography and Electrospray Ionization Mass Spectrometry, J. Agric. Food Chem., 2008, 56, 5758-5764.

8 E. B. Rodriguez, M. L. Vidallon, D. J. Mendoza and C. T. Reyes, Health-promoting bioactivities of betalains from red dragon fruit (Hylocereus polyrhizus (Weber) Britton and Rose) peels as affected by carbohydrate encapsulation, J. Sci. Food Agric., 2016, 96, 4679-4689.

9 P. Esquivel, F. C. Stintzing and R. Carle, Phenolic compound profiles and their corresponding antioxidant Capacity of purple pitaya (Hylocereus sp.) genotypes, $Z$. Naturforsch., C: J. Biosci., 2007, 62, 636-644.

10 C. Gentile, L. Tesoriere, M. Allegra, M. A. Livrea and P. D'Alessio, Antioxidant betalains from cactus pear (Opuntia ficus-indica) inhibit endothelial ICAM-1 expression, Ann. N. Y. Acad. Sci., 2004, 1028, 481-486.

11 L. Tesoriere, A. Attanzio, M. Allegra and M. A. Livrea, Dietary indicaxanthin from cactus pear (Opuntia ficusindica L. Mill) fruit prevents eryptosis induced by oxysterols in a hypercholesterolaemia-relevant proportion and adhesion of human erythrocytes to endothelial cell layers, Br. J. Nutr., 2015, 114, 368-375.

12 B. Yang, F. Cao, H. Zhao, J. Zhang, B. Jiang and Q. Wu, Betanin ameliorates isoproterenol-induced acute myocardial infarction through iNOS, inflammation, oxidative stress-myeloperoxidase/low-density lipoprotein in rat, Int. J. Clin. Exp. Pathol., 2016, 9, 2777-2786. 
13 S. Asgary, M. R. Afshani, A. Sahebkar, M. Keshvari, M. Taheri, E. Jahanian, M. Rafieian-Kopaei, F. Malekian and N. Sarrafzadegan, Improvement of hypertension, endothelial function and systemic inflammation following short-term supplementation with red beet (Beta vulgaris L.) juice: a randomized crossover pilot study, J. Hum. Hypertens., 2016, 30, 627-632.

14 A. Jajja, A. Sutyarjoko, J. Lara, K. Rennie, K. Brandt, O. Qadir and M. Siervo, Beetroot supplementation lowers daily systolic blood pressure in older, overweight subjects, Nutr. Res., 2014, 34, 868-875.

15 N. Abd Hadi, M. Mohamad, M. A. K. Rohin and R. Mohd Yusof, Effects of red pitaya (Hylocereus polyrhizus) consumption on blood glucose level and lipid profile in type 2 diabetic subjects, Borneo Sci., 2013, 31, 113-129.

16 B. Grube, P. W. Chong, K. Z. Lau and H. D. Orzechowski, A natural fiber complex reduces body weight in the overweight and obese: a double-blind, randomized, placebocontrolled study, Obesity, 2013, 21, 58-64.

17 P. Lopez-Romero, E. Pichardo-Ontiveros, A. Avila-Nava, N. Vazquez-Manjarrez, A. R. Tovar, J. Pedraza-Chaverri and $\mathrm{N}$. Torres, The effect of nopal (Opuntia ficus indica) on postprandial blood glucose, incretins, and antioxidant activity in Mexican patients with type 2 diabetes after consumption of two different composition breakfasts, J. Acad. Nutr. Diet., 2014, 114, 1811-1818.

18 I. J. Onakpoya, J. O’Sullivan and C. J. Heneghan, The effect of cactus pear (Opuntia ficus-indica) on body weight and cardiovascular risk factors: a systematic review and metaanalysis of randomized clinical trials, Nutrition, 2015, 31, 640-646.

19 N. Poolsup, N. Suksomboon and N. J. Paw, Effect of dragon fruit on glycemic control in prediabetes and type 2 diabetes: A systematic review and meta-analysis, PLoS One, 2017, 12, e0184577.

20 A. Sandoo, J. J. C. S. V. van Zanten, G. S. Metsios, D. Carroll and G. D. Kitas, The endothelium and its role in regulating vascular tone, Open Cardiovasc. Med. J., 2010, 4, 302-312.

21 H. F. Langer and T. Chavakis, Leukocyte-endothelial interactions in inflammation, J. Cell. Mol. Med., 2009, 13, 12111220.

22 P. O. Bonetti, L. O. Lerman and A. Lerman, Endothelial Dysfunction, Arterioscler., Thromb., Vasc. Biol., 2003, 23, 168-175.

23 R. J. Widmer and A. Lerman, Endothelial dysfunction and cardiovascular disease, Glob. Cardiol. Sci. Pract., 2014, 2014, 291-308.

24 R. T. Ras, M. T. Streppel, R. Draijer and P. L. Zock, Flowmediated dilation and cardiovascular risk prediction: A systematic review with meta-analysis, Int. J. Cardiol., 2013, 168, 344-351.

25 A. Covic and D. Siriopol, Pulse Wave Velocity Ratio, Hypertension, 2015, 65, 289-290.

26 D. Tousoulis, C. Antoniades and C. Stefanadis, Evaluating endothelial function in humans: a guide to invasive and non-invasive techniques, Heart, 2005, 91, 553-558.
27 D. Schuler, R. Sansone, T. Freudenberger, A. RodriguezMateos, G. Weber, T. Y. Momma, C. Goy, J. Altschmied, J. Haendeler, J. W. Fischer, M. Kelm and C. Heiss, Measurement of endothelium-dependent vasodilation in mice-brief report, Arterioscler., Thromb., Vasc. Biol., 2014, 34, 2651-2657.

28 B. Yu, D. J. Xu, H. Sun, K. Yang and M. Luo, Comparative Analysis of Mechanical Properties of PWV, NO and Ascending Aorta between WHY Rats and SHR Rats, Acta Cardiol. Sin., 2015, 31, 429-435.

29 J. P. T. Higgins and S. Green, Cochrane Handbook for Systematic Reviews of Interventions Version 5.1.0, 2011.

30 D. Moher, A. Liberati, J. Tetzlaff and D. G. Altman, Preferred reporting items for systematic reviews and metaanalyses: the PRISMA statement, Br. Med. J., 2009, 339, b2535.

31 N. Blanch, P. M. Clifton and J. B. Keogh, A systematic review of vascular and endothelial function: effects of fruit, vegetable and potassium intake, Nutr., Metab. Cardiovasc. Dis., 2015, 25, 253-266.

32 H. E. Fabela-Illescas, R. Avila-Dominguez, A. HernandezPacheco, J. A. A. Ortega and G. Betanzos-Cabrera, Effect of a Beverage Made from Cactus Pear (Nopalea Cochenillifera (L) Salm-Dyck) in a Rural Population of Hidalgo, Mexico; a Pilot Clinical Trial, Nutr. Hosp., 2015, 32, 2710-2714.

33 Y. C. Wang, Z. P. Qi, Z. Z. Liu, T. Li, H. X. Cui, B. Q. Wang and N. Chi, Therapeutic effects and mechanisms of Opuntia dillenii Haw on atherosclerosis of rats. [Chinese], Yaoxue Xuebao, 2015, 50, 453-458.

34 K. R. Anand Swarup, M. A. Sattar, N. A. Abdullah, M. H. Abdulla, I. M. Salman, H. A. Rathore and E. J. Johns, Effect of dragon fruit extract on oxidative stress and aortic stiffness in streptozotocin-induced diabetes in rats, Pharmacogn. Res., 2010, 2, 31-35.

35 M. Bakour, N. Al-Waili, R. El-Haskoury, N. El-Menyiy, T. AlWaili, A. Al-Waili and B. Lyoussi, Comparison of hypotensive, diuretic and renal effects between cladodes of Opuntia ficus-indica and furosemide, Asian Pac. J. Trop. Med., 2017, 10, 900-906.

36 Y. Cardenas, M. Rios-Silva, M. Huerta, M. Lopez, J. BricioBarrios, M. Ortiz-Mesina, Z. Urzua, A. Saavedra-Molina and X. Trujillo, The Comparative Effect of Nopal and Mucilage in Metabolic Parameters in Rats with a High-Fructose Diet, J. Med. Food, 2019, 22, 538-541.

37 N. E. Harrat, S. Louala, F. Bensalah, F. Affane, H. Chekkal and M. Lamri-Senhadji, Anti-hypertensive, anti-diabetic, hypocholesterolemic and antioxidant properties of prickly pear nopalitos in type 2 diabetic rats fed a high-fat diet, Nutr. Food Sci., 2019, 49, 476-490.

38 R. Saleem, M. Ahmad, A. Azmat, S. I. Ahmad, Z. Faizi, L. Abidi and S. Faizi, Hypotensive activity, toxicology and histopathology of opuntioside-I and methanolic extract of Opuntia dillenii, Biol. Pharm. Bull., 2005, 28, 1844-1851.

39 N. S. Ramli, L. Brown, P. Ismail and A. Rahmat, Effects of red pitaya juice supplementation on cardiovascular and hepatic changes in high-carbohydrate, high-fat diet- 
induced metabolic syndrome rats, BMC Complementary Altern. Med., 2014, 14, 189.

40 P. S. Oh and K. T. Lim, Glycoprotein (90 kDa) isolated from Opuntia ficus-indica var. saboten MAKINO lowers plasma lipid level through scavenging of intracellular radicals in Triton WR-1339-induced mice, Biol. Pharm. Bull., 2006, 29, 1391-1396.

41 L. Y. Zhao, W. Huang, Q. X. Yuan, J. Cheng, Z. C. Huang, L. J. Ouyang and F. H. Zeng, Hypolipidaemic effects and mechanisms of the main component of Opuntia dillenii Haw. polysaccharides in high-fat emulsion-induced hyperlipidaemic rats, Food Chem., 2012, 134, 964-971.

42 A. Khouloud, S. Abedelmalek, H. Chtourou and N. Souissi, The effect of Opuntia ficus-indica juice supplementation on oxidative stress, cardiovascular parameters, and biochemical markers following yo-yo Intermittent recovery test, Food Sci. Nutr., 2018, 6, 259-268.

43 P. Rahimi, S. A. Mesbah-Namin, A. Ostadrahimi, S. Abedimanesh, A. Separham and M. Asghary Jafarabadi, Effects of betalains on atherogenic risk factors in patients with atherosclerotic cardiovascular disease, Food Funct., 2019, 10, 8286-8297.

44 J. Wiese, S. McPherson, M. C. Odden and M. G. Shlipak, Effect of Opuntia ficus indica on symptoms of the alcohol hangover, Arch. Intern. Med., 2004, 164, 1334-1340.

45 L. Schmitt, J. P. Fouillot, G. Nicolet and A. Midol, Opuntia ficus indica increases heart-rate variability in high-level athletes, Int. J. Sport Nutr. Exercise Metab., 2008, 18, 169-178.

46 K. Van Proeyen, M. Ramaekers, I. Pischel and P. Hespel, Opuntia ficus-indica ingestion stimulates peripheral dispo- sal of oral glucose before and after exercise in healthy men, Int. J. Sport Nutr. Exercise Metab., 2012, 22, 284-291.

47 I. Dhananjayan, S. Kathiroli, S. Subramani and V. Veerasamy, Ameliorating effect of betanin, a natural chromoalkaloid by modulating hepatic carbohydrate metabolic enzyme activities and glycogen content in streptozotocin - nicotinamide induced experimental rats, Biomed. Pharmacother., 2017, 88, 1069-1079.

48 A. Rodriguez-Mateos, M. Hezel, H. Aydin, M. Kelm, J. O. Lundberg, E. Weitzberg, J. P. E. Spencer and C. Heiss, Interactions between cocoa flavanols and inorganic nitrate: Additive effects on endothelial function at achievable dietary amounts, Free Radical Biol. Med., 2015, 80, 121-128.

49 T. Sawicki, J. Topolska, E. Romaszko and W. Wiczkowski, Profile and Content of Betalains in Plasma and Urine of Volunteers after Long-Term Exposure to Fermented Red Beet Juice, J. Agric. Food Chem., 2018, 66, 4155-4163.

50 A. Avolio, M. Butlin, Y.-Y. Liu, K. Viegas, B. Avadhanam and G. Lindesay, Regulation of arterial stiffness: Cellular, molecular and neurogenic mechanisms, Artery Res., 2011, 5, 122-127.

51 D. A. Hobbs, N. Kaffa, T. W. George, L. Methven and J. A. Lovegrove, Blood pressure-lowering effects of beetroot juice and novel beetroot-enriched bread products in normotensive male subjects, Br. J. Nutr., 2012, 108, 20662074.

52 S. Lidder and A. J. Webb, Vascular effects of dietary nitrate (as found in green leafy vegetables and beetroot) via the nitrate-nitrite-nitric oxide pathway, Br. J. Clin. Pharmacol., 2013, 75, 677-696. 